\title{
PERSONA, ADQUISICIÓN DE LA L2 Y NUEVAS TECNOLOGÍAS
}

\author{
Felipe Alcántara Iglesias* \\ Universidad de La Rioja
}

\begin{abstract}
Although the notion that individual internal factors play an essential role in language learning, most of the acquisition/learning research has been done on aspects concerned with techniques and design of environmental changes of the external factors. Yet what belongs to the intra-individual field can strongly alter the final results of a good language teaching programme. To analyse some of the advantages that modern technology can offer to language teaching we use concepts from very different subject fields. This is the case of the psychoanalitic concept of "persona" which, although apparently unrelated, can serve to make explicit that the Vygostkian harmony between intraindividual and inter-individual factors is paramount when it comes to make a good use of the new technological advances for the teaching of languages.

RESUMEN. Los estudios sobre el aprendizaje verbal no siempre dan debida cuenta de las circunstancias internas al sujeto que aprende. Una buena articulación de las circunstancias externas para potenciar el aprendizaje puede no resultar productiva si no se armoniza bien con los elementos intra-individuales del sujeto. Ofrecemos aquí una reflexión sobre aspectos representativos de estos dos ámbitos que, aunque bien diferentes en su concepción, los estimamos convergentes en su fin. Analizamos el concepto psicoanalítico de "persona", la lengua, su aprendizaje y las nuevas tecnologías.
\end{abstract}

\section{Introducción}

El hombre vive en un universo extraordinariamente complejo, a pesar de ello, los avances que una generación consigue sobre lo realizado por generaciones anteriores es verdaderamente sorprendente. Sin embargo, el progreso del conocimiento en los tiempos modernos se ha producido fundamentalmente sobre el universo exterior al hombre y ha sido posible gracias a la especialización. Las limitaciones de la mente humana obligan al hombre a fragmentar el objeto de su conocimiento en partes abarcables,

\footnotetext{
* Profesor de Filología Inglesa. Departamento de Filologías Modernas.
} 
esto puede tener como consecuencia no deseable el aislamiento del saber resultante del estudio de esas fracciones si no se realizan esfuerzos para lograr posibles síntesis con los resultados obtenidos, tanto por esfuerzos anteriores, como con los logros resultantes de actuaciones diversas.

A pesar del enorme progreso del conocimiento de los fenómenos del universo físico -exterior al ser humano-; sobre otros fenómenos -los interiores al hombre- los mentales, el avance no ha sido tan espectacular, desafortunadamente. Quizás sean estos últimos los que, a nuestro entender, requieran más del concurso de todas las perspectivas posibles para poder conseguir aunque solamente sea una comprensión limitada. La lengua además de producir una conducta observable, es una parte fundamental de la mente, de sus procesos y de su representación. En el estudio de la lengua, pensamos, no se puede disociar al sujeto hablante, de sus estados internos, de la influencia del medio interior y exterior al mismo. Cuando se trata del estudio de la adquisición de otra lengua, con frecuencia la metodología en curso, el paradigma dominante, propicia formas de proceder propias de las metodologías empleadas en el avance del conocimiento científico del universo físico, lo exterior al hombre; de forma que se incurre, quizás, en una fragmentación excesiva, al tiempo que se objetiva el fenómeno verbal, olvidándose con frecuencia aquello que hace referencia a lo interior al sujeto que aprende. De esta manera, se producen líneas investigadoras que entienden las manifestaciones verbales como fenómenos arbitrarios e independientes; al tiempo que su aprendizaje aparece desligado de los estados internos del alumno.

En este trabajo pretendemos analizar algunos aspectos de ese interior del sujeto y relacionar aquellos con el proceso adquisitivo de la L2. En las páginas finales establecemos una vinculación entre las posibles consecuencias de nuestros razonamientos y aquella parte del avance del conocimiento sobre el universo exterior al hombre: el científico-técnico; vemos desde nuestra perspectiva la relación que el progreso en el campo de la ciencia de la información tiene con la enseñanza y adquisición de la L2 y sugerimos, también, la posible aplicación de los medios disponibles a determinados problemas internos del alumno.

\section{La persona}

El concepto de persona, tal como suele usarse en el psicoanálisis ${ }^{1}$, nos parece susceptible de ser incorporado dentro del marco conceptual en el que se desarrollan

1. El concepto de persona es usado fundamentalmente por el psicoanálisis como expresión de la configuración individual con la realidad externa. Jung utilizó este término [persona] para caracterizar la expresión del impulso arquetípico hacia la conformidad y la realidad externa. La persona es la máscara que cubre la personalidad del individuo; es la cara que éste presenta al mundo externo. En sueños, la persona es representada en forma de imágenes de vestidos y como problemas que se refieren a vestidos. 
los estudios sobre la adquisición de la segunda lengua (L2). Entendemos que su consideración puede resultar útil para explicar el proceso comunicativo sobre el cual gira actualmente $^{2}$ la enseñanza de la lengua. Hoy que las ciencias cognitivas se preocupan por el estudio de la estructura de la acción, nos parece que el uso del concepto de persona puede facilitar la articulación del aspecto individual dentro de la acción verbal y puede ayudar a entender mejor la relación dialéctica que se establece entre lo interior y lo exterior al sujeto; la semantización de lo social, en cuanto medio y objeto de la actividad verbal: la comunicación.

Habida cuenta de que la actividad verbal representa siempre una tensión entre el yo y el otro, la persona nos parece que es causa y efecto de esta interrelación a través de la expresión verbal fundamentalmente; y el aprendizaje de una nueva forma de expresión verbal puede implicar, o requerir cambios en la propia persona, de aquí la conveniencia de su consideración en el estudio de la adquisición de la L2. Por otra parte, la relación entre la psicología clínica y la enseñanza de la L2 no es un hecho singular; como se sabe tanto Curran, como Lozanov desarrollaron sus peculiares enfoques sobre la enseñanza de otras lenguas desde presupuestos propios de la experiencia y el conocimiento clínico. Este es el caso, también, de Guiora, sobre cuyas ideas comentaremos a continuación.

$\mathrm{Si}$, además, entendemos que el lenguaje verbal es parte consubstancial de la constitución del yo y de su mente autoconsciente, como desde un punto de vista bien diferente indica Popper ${ }^{3}$, es fácilmente asumible que una nueva forma de expresión suponga, al menos, una nueva manera de manifestar y, por lo tanto, de estructurar la persona hacia los otros -como viene en resumen a decir Guiora ${ }^{4}$. Consecuentemente, cabe suponer también, que la personalidad del que aprende debe asumir el esfuerzo que supone el proceso organizativo de las formas de expresión individuales en la nueva lengua; lo que puede conmover aspectos íntimos de su estructura personal, tales como su emotividad, la conciencia de su yo, la actitud para sí y para los otros, etc.

\section{II}

Siempre se ha considerado que ciertos elementos que forman la personalidad del estudiante mostraban un determinado grado de incidencia -positiva o negativa- sobre

2. Aunque, en el campo de la didáctica al menos, se tiende a pensar en "lo comunicativo" como alternativa actual a orientaciones pasadas, la novedad del enfoque comunicativo no es tal. Ya a comienzos de siglo habían insistido algunos psicolingüistas en la necesidad de tomar como unidad análisis el diálogo, en lugar de la palabra, o la frase aislada, y se había desarrollado un volumen teórico considerable al respecto. Ver, por ejemplo, VOLOSINOV, V.N.

Marksizm I Filosofija Jazyka. Leningrado, 1930. (trad. ingl. Marxism and the Philosophy of Language, 1973, Londres, Seminar Press).

3. POPPER, K. Búsqueda sin término, Madrid, Tecnos, 1977.

4. GUIORA, A.Z. "The dialectic of language acquisition". Language Learning, 5, 1983 
los resultados del aprendizaje de una nueva lengua 5 . Pero una consideración amplia de las razones por las cuales se triunfa, o se fracasa, en el aprendizaje de la nueva lengua, o la posible explicación de ciertas dificultades extremas -como es el caso de la pronunciación- exigen un concurso multidisciplinar desde aquellos campos del saber en los que pueda encontrarse algún elemento relacionable con estos problemas; lo que no es tarea fácil, por la marcada tendencia a la especialización de la tarea investigadora.

Sin embargo, contemplar al alumno como una unidad psicosomática, en relación con las otras unidades que componen el resto de los compañeros y la del profesor, parece, al menos intuitivamente, ciertamente prometedor. Entender, consecuentemente, que en el transcurso del aprendizaje los sujetos se encuentran inmersos en un proceso integral que puede producir una posible reestructuración de los elementos que constituyen la persona, como consecuencia de los cambios que impone el nuevo medio expresivo, no es, no obstante, tan frecuente. Sin embargo, este tema ya fue tratado por autores como Brachfeld en 1936, o Stengel en 19396; durante las tres décadas siguientes las cuestiones de índole psicológica se enfocan prioritariamente desde las bases de la psicología de la conducta y se producen modelos generales sobre el aprendizaje de la L2 en el que esta ausente toda variación de tipo individual. Sin embargo, ya en 1965 el grupo de investigación de Guiora ${ }^{7}$, compuesto por psicólogos, psiquiatras, lingüistas y psicolingüistas publicaron su primer estudio sobre la intuición, la inferencia y la empatía ${ }^{8}$, desde unos supuestos teóricos diferentes a los de la teoría de la conducta. Quizás por ello no fueron acogidos debidamente. Hoy, que el paradigma de la conducta ha dejado de ser dominante, puede ser conveniente su reconsideración.

Por medio de un método de observación al que denomina "investigación transposicional" trataba Guiora de establecer una correlación entre empatía y nivel de pronunciación en la L2, así como la configuración de los roles y su manifestación verbal en forma de registros lingüísticos. De esta manera, Guiora quería probar que el nivel de pronunciación adquirido por el sujeto está en relación directa con su empatía; y que ambas están fuertemente influenciadas por el mismo proceso subyacente: lo que denomina la "permeabilidad de las fronteras del ego" del sujeto. Además del "ego corporal", Guiora

5. Por ejemplo, Morrison, A.V. 1961 Personality and underachievement in foreign language learning... Reseach Report. U.S. Department of H. E. and Welfare. C N. CE2-14-004, comprueba lo que era, y es, un sentimiento generalmente compartido por los profesionales de la enseñanza de la lengua: ciertas configuraciones de la personalidad, como pueden ser aquellas que se manifiestan en una mayor sociabilidad y extroversión, contribuyen positivamente a un mejor y más rápido aprendizaje de una L2.

6. Cfr. STERN, H, H. Fundamental Concepts of Language Teaching. Oxford, O.U.P. 1983, cap. XV.

7. GUIORA, A.Z., R.K. BOLIN, C.E. DUTTON y B. MEER "Intuition: a preliminary statement" Psychiatric Quarterly Supplement, 39, 1965.

8. Así definen la empatía:

"[...] a process of comprehending in which a temporary fusion of the self-object boundaries, as in the earliest pattern of object relation, permits an immediate emotional apprehension of the affective experience of the other, this sensing being used by the cognitive functions to gain understanding of the other"

(GUIORA y otros, "Intuition", op. cit., pág. 165.) 
propone el "ego verbal"; el primero viene a ser la auto-representación física y el segundo la verbal. Siendo ambos producto del desarrollo de la persona del sujeto, sus límites tienden a estabilizarse y se hacen rígidos e impermeables a partir de una determinada edad, que coincide sensiblemente con la pubertad. Desde este punto de vista Guiora ofrece una explicación diferente a la de la plasticidad cerebral en lo que respecta a la dificultad de conseguir una buena pronunciación en los alumnos adultos.

Es una idea ampliamente compartida que el nivel de pronunciación no guarda relación con la motivación, el grado de instrucción ni la inteligencia del alumno. Los resultados de los trabajos de Guiora confirman experimentalmente estos extremos 9 . También ofrecen evidencias que permiten suponer una vinculación estrecha entre la auto-representación y la expresión verbal, lo que Guiora expresa de la siguiente forma:

Ridicule the way I sound, my dialect, or my attempts at pronouncing French and you will have ridiculed me. Ask me to change the way I sound and you ask me to change myself. To speak a second language authentically is to take on a new identity ${ }^{10}$.

La vinculación entre la lengua y la persona es, al menos intuitivamente, ampliamente reconocida, sin embargo, lo que las tesis de Guiora aportan de nuevo es la dirección en que esta vinculación debe establecerse: lo que en la investigación precedente se había establecido con los nombres genéricos de "factores internos", o "variables afectivas". Pero estos factores internos difícilmente pueden concebirse en un marco teórico que entiende al sujeto como causa y efecto de su propia configuración interna. Tampoco ha dado mucho de sí las posiciones opuestas que, como la conductista, han tratado de explicar la conducta verbal del sujeto en función de determinadas secuencias de estados del medio exterior.

Ya a principios de siglo Vygotsky indicaba la conveniencia de buscar lo intraindividual en lo inter-individual. Sin embargo la interacción entre estos dos campos no debe entenderse en términos de un sujeto determinado por los estímulos exteriores, sino como una entidad extraordinariamente compleja en constante interacción con su medio exterior, que tiene fundamentalmente una naturaleza social. La relación que

9. "Each one of us has a set of pronunciation habits which are invariable unless factors in our make up can make us more flexible, unless we have the ability to step outside of our 'language shoes' and expand our identity in some way [...] this ability do shed our native language pronunciation habits and temporarily adopt a different pronunciation is closely related to empathic capacity [...] Our conclusion was that we had indeed shown that empathic ability as measured by the MME is an important variable in the ability of authentically pronounce a second language." GUIORA, A.Z. R.C.L. BRANNON, C.Y. DULL, T. SCOVEL, "The effects of experimentally induced changes in ego states on pronunciation ability in a second language" Comprehensive Psychiatry, 13, 1972, pág. 52.

10. GUIORA, A, M.PALUSZNY, B. BEIT-HALLAHMI, J.C. CATFORD, R. COOLEY Y C. YODER. "Language and person. Studies in language behavior", Language Learning, 25, 1975, pág. 48. 
Guiora establece entre la forma de hablar y los límites del ego está basada, precisamente, en la forma en que la persona aparece ante los otros: su auto-representación. De manera que cabe entender, que los cambios en la imagen verbal que presentamos a los otros - o lo que Guiora denomina "ego verbal"- deben ser tolerados por un grado de elasticidad suficiente de los rasgos que podemos considerar definidores de la personalidad. Así interpretamos el trabajo de Guiora en el que establece una correlación entre la ingestión de alcohol y la mejora de la pronunciación en su experimento de 197211.

Por otra parte, si el origen de lo intra-subjetivo se puede encontrar en la relación con los otros, si el centro de nuestro interés está localizado en el proceso verbal, en la relación comunicativa, parece conveniente que, al estudiar esta relación, se tengan en cuenta otros elementos prioritarios -además del estudio del empleo de la lengua. Entendemos que una de estas prioridades bien puede ser la vinculación entre la persona y la lengua. Sin embargo, el marco conceptual dominante desde el que generalmente se estudia la adquisición de la L2 no ha dejado el suficiente espacio teórico para ello. No obstante, esta necesidad parece haber estado presente ya en los inicios del movimiento comunicativo. Por ejemplo Brown ${ }^{12}$, en una perspectiva para los estudios sobre la L2, a mediados de los setenta reclama la necesidad de una mayor atención a las "variables afectivas" que intervienen en el aprendizaje de lenguas extranjeras; esa atención se evidenciará pronto con un considerable aumento de las publicaciones sobre estos temas ${ }^{13}$. Ahora bien, al incorporar el factor afectivo desde una perspectiva psicológica que entiende la cognición como causa de los cambios producidos en el medio exterior, se hace con la idea de que el flujo input/output encuentre la menores constricciones posibles dentro del sujeto. Para ello se recomienda que, para facilitar el aprendizaje verbal, es conveniente amenizar la tarea, realizar actividades de relajación e infantilización del alumno., etc. En definitiva, procedimientos orientados a preparar al sujeto para la recepción de un objeto extraño: la lengua que está aprendiendo, mediante técnicas generales y altamente estandarizadas que llevan en sí mismas la

11. El experimento consistió en hacer ingerir ciertas cantidades de alcohol $(0,1,1,5,2$, ó 3 onzas [1 oz. $=28,35 \mathrm{ml}$.]) a 87 sujetos varones distribuidas en relación con el peso de cada uno. La mitad del grupo ingirió también algo de comida dulce, realizándose el experimento diez minutos después de la ingestión del alcohol. Se suministraron los test "Digit Symbol Test"-un sub-test de el "Weschler Adult Intelligence Scale", así como el "Standard Thai Procedure" diseñado por Guiora para medir la habilidad en la pronunciación de una L2. Se encontró una mejora altamente significativa en los niveles de pronunciación en los sujetos del grupo que, habiendo comido algo dulce habían bebido 1,5 oz de alcohol, mientras en que los del mismo grupo que habían ingerido cantidades mayores su habilidad decreció. El grupo que no había comido no mostró efectos significativos asociables a las cantidades de alcohol ingeridas. Para mayor detalle cfr. GUIORA y otros, "Persona”, op. cit.1975, pág. 52 y ss.

12. BROWN, D.H. "Affective variables in second language acquisition", Language Learning, 23. 1973.

13. Para una buena relación de este tipo de publicaciones, ver: STERN, H.H. Fundamental Concepts of Language Teaching, Oxford, O.U.P.,1983, cap. XVII. 
desconsideración más absoluta de las sutiles relaciones existentes entre la persona y la lengua.

\section{III}

Un somero análisis de la ontogénesis del lenguaje desde el punto de vista que proponemos, nos revela de inmediato la íntima relación entre el ser humano y su expresión verbal. Tengamos en cuenta que, desde los primeros momentos de la vida del niño, se desarrolla un sistema específico de relación entre el recién nacido y sus padres. Es tan importante la influencia de este sistema sobre la futura organización que puedan tener sus formas de relación interpersonal -y por lo tanto intra-personalporque es en ese momento cuando se "generan ya los embriones del universo expresivo en el que vive el ser humano", dice Diatkine14. Porque estos no tiene su origen en el signo para el sujeto productor del mismo; sino en lo que puede considerarse un signo para los sujetos receptores: los padres. Son ellos quienes mediante la interpretación comienzan a acercar al umbral del signo lo que no es más que un acto reflejo genéticamente adquirido. De forma que, la transición a otras formas significativas más elaboradas y sistemáticas, como puede ser la aparición del "gesto", en el sentido de G.H. Mead15, lo justifica Diatkine ${ }^{16}$ gracias a lo que denomina "ilusión anticipatoria de la madre". Esto nos llevaría a concluir que, una determinada expresión por parte del niño se convierte en signo gracias a esa ilusión anticipatoria de la madre -o cuidadores de éste. Se trata de la atribución de significado ejercitando la intuición, por medio de un acto comunicativo establecido sobre una base afectiva. Obviamente, los signos producidos por el niño no son, en un principio, tales signos para él; sino sólo para sus cuidadores. Además, el niño no recibe una respuesta inequívoca a la que asociar un significado. Por el contrario, lo que parece producirse, es un intercambio expresivo con un considerable grado de incertidumbre ${ }^{17} \mathrm{y}$, en todo caso, desde luego de una manera mucho menos mecánica y delimitada de la que se puede establecer desde el marco teórico del asociacionismo.

This unbalanced exchange in which illusion and projection are foremost (i.e. particular form of knowledge where psychological reality is more important than any other mode of reference to reality) is rather representative of an entire

14. DIATKINE, R. "The child in communication within different social surroundings" International Journal of Psycholinguistics, 2, 1980.

15. MEAD, G.H. Mind, Self and Society. University of Chicago Press, Chicago, 1934, (traduc. esp. Espíritu, persona y sociedad. Paidós, Barcelona 1963.

16. DIATKINE, "The child", op.cit.

17. BRUNER, J. "The role of dialogue in language acquisition". En Sinclair, Jarvilla y Levelt (eds.) The Child Conception of Language. Springer Verlag, 1978. 
series of successive systems of communication where misconceptions play as important a role as correct decodings ${ }^{18}$

Si lo que perseguimos es un mejor entendimiento de la acción comunicativa, no podemos olvidar que la primera actividad comunicativa, según esta autora, supone la introducción del niño en el universo de la afectividad; lo que se realiza, fundamentalmente, a través de los contactos visuales como forma de transmitir estados emocionales, pero también mediante la creación de un telón en el que la persona que se relaciona con el niño proyecta su "ego ideal": "If the person evokes sufficiently powerful interest on the part of the child to provide a backdrop on which his ego ideal can be projected"19. Posteriormente, y mediante un fenómeno que entendemos similar a lo que Lacan llama "el efecto de espejo", se va consolidando una forma elemental de comunicación basada en la transmisión de afecto y en la regulación de la conducta por parte de los adultos. Esto dará lugar al desarrollo de una lengua propia, idosincrática de cada niño, como establece Halliday ${ }^{20}$. Finalmente se producirá la paulatina adopción de la lengua de los adultos por parte de aquél. Pero, para poder adquirir las formas de expresión verbal adultas, el niño tiene que emprender un largo camino en el que irá asimilando, no solo aquellas expresiones que se dirigen a él de una manera directa, sino también aquellas que, simplemente, son producidas en su presencia y con otro destinatario. Este último aspecto nos alerta contra la sobre utilización de las actividades interactivas en el aula de idiomas, pues la vía interactiva no parece ser el único camino de la adquisición de la lengua -como hoy se pretende por parte de algunos autores ${ }^{21}$.

\section{IV}

En su estudio sobre la asimilación de los términos léxicos Miller y Gildea 22 estiman que desde que se inicia en la adquisición de la lengua de los adultos -cuando "empieza a hablar"- el niño aprende una media de más de diez palabras nuevas cada día (diez vocablos de raíz distinta, los derivados no se cuentan)23. estos mismos

18. DIATKINE,’The child”, op. cit., pág. 139.

19. DIATKINE, ibid.

20. HALLIDAY, M.A.K Learning How to Mean. Londres, Arnold, 1975.

21. Por ejemplo, PICA, T. "Interlanguage ajustments of NS-NSS negotiated interaction". Language Learning, 11988.

22. MILLER, G.A. Y GILDEA, P.M. “Como aprenden las palabras los niños”. Comunidad Científica, 3, 1988.

23. MILLER Y GILDEA hacen el siguiente cómputo: “...el vocabulario de lectura de un bachiller superior, por ejemplo, constará de alrededor de unas 40.000 palabras. Si se consideran también palabras todos los nombres propios de personas y lugares y todas las expresiones idiomáticas, deberá doblarse esta cantidad. La cifra habla pro sí sola de la capacidad infantil de aprender vocabulario. Si el bachiller promedio cuenta 17 años de edad, esas 80.000 palabras se habrán aprendido en 16 años, por lo que el infante promedio aprende a razón de 5.000 palabras por año, es decir, 13 diarias. [...] . Nadie les enseña diariamente a 
autores reconocen en su trabajo que "se conocen mal los mecanismos de ese logro". Por supuesto, no lo hacen memorizando entradas de diccionario ${ }^{24}$. Cuando el niño alcanza los primeros grados de escolaridad, reconocen estos autores que para desarrollar el lenguaje escrito se apoya en las palabras que ya ha aprendido verbalmente pero, añaden:

El aprendizaje de un nuevo vocablo requiere tal claridad conceptual y ejercicio fonológico que no hay horas de clase suficientes para enseñar de ese modo más de 100 o 200 palabras por año. El aprendizaje (sic.) le saca tanta ventaja a la enseñanza (se aprenden una 5.000 palabras cada año y sólo se enseñan unas 200) que resulta difícil eludir la pregunta: cómo se las arreglan los escolares para aprender mucho más de lo que se les enseña?25.

Sin pretender tener la respuesta a tan complicada pregunta, sí cabe, sin embargo, hacer una consideración sobre el sentido que puede atribuirse a las palabras anteriores. Difícilmente la asimilación de una lengua, ni siquiera de un componente de la misma como es su parte léxica, puede ser producto del "aprendizaje" tal como parece desprenderse del trabajo de estos autores; esto es, como producto de una conceptualización y de una enseñanza directa. Parece más razonable suponer que una buena parte de lo que se aprende es una especie de subproducto de la actividad comunicativa -la práctica de la escritura sería una variedad más. En otras palabras, cuando el objeto de la actividad no cae dentro de la actividad verbal; sino que pertenece a una actividad más general -o de orden superior. En este sentido cabría suponer que en lugar de una conceptualización, que requiere el concurso de la conciencia, se trata de una especie de aprendizaje funcional de los medios expresivos, cuyas unidades cognitivas no tienen por qué coincidir siempre con las unidades léxicas -por ejemplo. Con el propósito de establecer una diferencia similar, se usa la dicotomía "adquisición"/“aprendizaje", establecida por Krashen y hoy generalmente admitida. No es tan general el acuerdo entre los investigadores de la adquisición de la L2 acerca de que tal distinción deba implicar en el sujeto una predisposición psicológica diferenciada en ambos casos. De tal forma, que en lugar de basar la diferencia entre un proceso cognitivo y otro en la dicotomía consciente/inconsciente -lo que puede parecer una generalización excesi$v^{26}$ - sería deseable articular la forma y condiciones en las que el sujeto enfoca una u otra $\operatorname{actividad}^{27}$.

los niños un promedio de 13 o más palabras. Deben tener un talento especial para ese tipo de aprendizaje". MILLER Y GILDEA “Como aprenden”, op.cit., pág. 80.

24. MILLER Y GILDEA, “Como aprenden”, op. cit., pág. 80.

25. MILLER Y GILDEA, “Como aprenden”, op. cit., pág. 82.

26. Ver: GREGG, K.R. “Krashen's monitor and Occam's razor”.Applied Linguistics, 2, 1984 para un detallado análisis crítico de la teoria del "monitor" de Khrasen.

27. No han faltado manifestaciones sobre esta necesidad incluso dentro de mismo marco teórico del que surge la teoría de Krashen. El propio Chomsky (On the Generative Enterprise, Drodrech, The Netherlands, Foris, 1982, pág.69) lo expresa de la siguiente manera: 
Guiora se define por una posición de complementariedad. Al entender que la totalidad de la persona está implicada en ambos procesos -el "adquisitivo" y el de "aprendizaje"- reclama el valor trasposicional y heurístico que el estudio de la adquisición de la L2 puede tener sobre el conjunto cognitivo y afectivo de la conducta humana:

One should not treat the acquisition of native language and foreign language as two independent systems, but rather as complementary aspects of one basic cognitive-affective scheme, interacting and conflicting with each other in a variety of ways. Moreover, an illumination of the psychological processes impinging on one can shed light, in a reciprocal way, on the other ${ }^{28}$.

Pero:

..if the acquisition of first language is to serve as a model for the acquisition of a foreign language, it will be the psychodynamic and cultural dimensions of that process rather than its formal elements, that will prove to be heuristic 29.

Para ello parece de nuevo conveniente concebir al sujeto de aprendizaje de forma integral, en lugar de como mero sujeto cognoscente. Se podrían así analizar mejor evidencias como la que Miller y Gildea indican. Del mismo modo, se requiere también un análisis distinto del objeto: la lengua, contemplada como algo más ligado al ser humano y a su relación con los "otros". Dice Diatkine ${ }^{30}$ con respecto a la "ilusión anticipatoria" anteriormente citada que:

The child uses the words that are not addressed to him more than those that are, through a process of identification that is a response to the parents' anticipatory illusion.

This last point introduces the relationship between language communication and imaginary language, a relationship which leads, in turn to written language.

De forma que, en la actividad verbal -tanto en su faceta ejecutiva como perceptiva- el sujeto tiene presente, aunque no sea siempre de forma consciente, lo que Lacan denomina "el otro"31; y por lo tanto, su auto-representación ante él mediante su forma

\footnotetext{
"It is absolutely suicidal for a field to define itself the way psychology of language almost invariably does, as dealing with processes but not with the structures that might enter into them, or to deal with the observed stages of growth and development, but not with the systems that underlie them".

28. GUIORA, "The dialectic", op.cit., pág.3.

29. GUIORA, "The dialectic", op.cit., pág. 10.

30. DIATKINE, "The child", op. cit., pág. 140.

31. "La forma bajo la cual el lenguaje se expresa define por ella misma la subjetividad. Dice: 'Irás por aquí, y cuando veas esto, tomarás por allá'. Dicho de otra manera, se refiere al discurso del otro. Está
} 
de expresión verbal. Persona y lengua aparecen de nuevo, desde su punto de vista, íntimamente ligadas.

\section{$\mathbf{V}$}

Desde una perspectiva distinta, la sociolingüística, se evidencia igualmente esta vinculación. Así expresa Halliday la intervención del lenguaje verbal en la constitución de la persona:

Like the individual in many other species, he [the individual human organism] is destined to become of a group; but unlike those of all other species, he achieves this -not wholly, but critically- through language [...] it is largely the linguistic interchange with the group that determines the status of the individuals and shapes them as persons ${ }^{32}$

Podemos ver que la sociedad, en cuanto unidad de análisis superior al grupo, está constituida por diversos tipos de relaciones, las que -a su vez- definen los distintos papeles sociales. De manera que, el hecho de convertirse en miembro de una determinada sociedad trae consigo una determinada capacidad de ejecución de un conjunto de papeles sociales; y la manera de manifestarse en su ejecución puede considerarse como un reflejo de la personalidad. La lengua, de nuevo, es mediadora y condición necesaria para la modelación de la personalidad individual, por lo que Halliday define a ésta como un "role complex"33

Si nos desplazamos de nuevo a un nivel más bajo de análisis, el del sujeto de actividad verbal, son interesantes las siguientes palabras de Guiora:

Language, native language, is the very lifeblood of human self-awareness, it is the carrier of identity, the safe repository of a vast array of affective and cognitive templates making up the total web of personality. It encompasses what we are, and limits what we are not. If one wants to wax poetic, it is a divine gift. It is a divine gift because we don't have to do much of anything to acquire it, to use it, to clothe ourselves in it ${ }^{34}$.

envuelto como tal en la más alta función de la palabra, por cuanto compromete a su autor al investir a su destinatario con una realidad nueva, [...] es en la intersubjetividad del 'nosotros' que asume, en la que se mide en un lenguaje su valor de palabra.

[...] Lo que busco en la palabra es la respuesta del otro, Lo que me constituye como sujeto es mi pregunta. Para hacerme reconocer del otro, no profiero lo que fue sino con vistas a lo que será. Para encontrarlo, lo llamo con un nombre que él debe asumir o rechazar para responderme.

Me identifico en el lenguaje, pero sólo perdiéndome en él como un objeto.” LACAN, Escritos, Méjico, Ed. Siglo XXI, 1981, pág. 117.

32. HALLIDAY, M.A.K. Language as Social Semiotic, Londres, Arnold, 1978, pág. 14.

33. ibid.

34. GUIORA,"The dialectic”, op. cit., pág. 30. 
Quizás Guiora vaya demasiado lejos al final de esta afirmación que nos evoca el “dictum" Heideggeriano "Die Sprache ist das Haus des Seins". En efecto, se puede considerar cada manifestación verbal como una entidad fenomenológica; y así es posible comparar los sistemas observables en cada actividad verbal con los que pueden derivarse de su significado, para poder detectar cómo sus características operan constrictivamente; tanto sobre la lengua, como sobre sus hablantes. Pero, el enfoque fenomenológico está justificado por razones metodológicas, como han hecho Guiora y sus colaboradores acerca de la hipótesis Whorf-Sapir ${ }^{35}$. Sin embargo, es difícil aceptar lo que hay detrás de la imagen del desarrollo del lenguaje como "regalo divino"36. La aparente facilidad con la que parece desarrollarse lo que etiquetamos como "adquisición de la L1", es ilusoria. Es bien sabido que se trata de un proceso largo y complejo, cuya realización es posible, porque se dan las condiciones sociales y orgánicas apropiadas para ello. Ya hemos visto hasta qué momento de la vida del niño se remontan los relaciones inter-personales con influencia sobre el desarrollo verbal: "el sistema de apoyo". Desde el punto de vista orgánico, en el niño, en cuanto ser vivo, y dadas las condiciones ambientales necesarias, se produce la autopoiesis ${ }^{37}$ : el acoplamiento estructural al medio. En el caso del ser humano, se trata de un medio inminentemente social, en el que el empleo de una o varias formas de relación verbal son imprescindibles. Como tal sistema autopoiético, el niño constituye una estructura plástica, cambiante por el medio -pero no determinada por él. Los cambios son de acoplamiento a las transformaciones del medio -entre ellas las verbales. Si no se produjera esa adaptación estructural, creemos que según dice Maturana ${ }^{38}$ de todos los seres vivos, peligraría su integridad; en su caso, la integridad o unidad como ente social: la persona.

La llamada adquisición de la L1 es pues posible por darse orgánica y socialmente las condiciones apropiadas para ello. Cuando estas circunstancias cambian, como es el caso de la adquisición de una nueva lengua por el individuo adulto, como el propio Guiora acepta, la dificultad se muestra en toda su extensión. A nuestro entender, lo

35. Ver, por ejemplo, BEIT-HALLAHMI y otros. "Grammatical gender and gender identity development: cross-cultural and cross-lingual implications." American Journal of Orthopsychiatry, 3 1974, sobre la influencia de una de las características lingüísticas de la L1 -la forma de marcar el género- sobre otra de desarrollo psicológico. El estudio en cuestión mostró que, aunque alrededor de los cinco años la influencia estaba neutralizada, antes de esta edad, la lengua nativa que hace distinciones de género más explícitas, desarrolla en sus hablantes de temprana edad la distinción de género más precozmente.

36. La aparente facilidad con que el niño adquiere la lengua es ilusoria. En condiciones similares de aprendizaje el niño demuestra su inferioridad con respecto al adulto y al adolescente en todos los aspectos, con excepción de la pronunciación (Ellis, 1987). Esto hace que en la investigación sobre el desarrollo del lenguaje sean cada vez mayormente aceptadas palabras como las siguientes:

"Para muchos [niños], la adquisición de la primera lengua constituye la mayor hazaña intelectual que realizarán en toda la vida” MILLER Y GILDEA, “Como aprenden”, op. cit. pág. 80.

37. Ver: ZELENY, M. (ed.) Autopoiesis, a Theory of the Living Organization, Nueva York, ElsevierNorth Holland, 1978.

38. MATURANA, H. y F. VARELA, Autopoiesis and Cognition, Dordrecht, Dreiden, 1980. 
que este autor expresa con palabras tan evocadoras sobre la vinculación entre el ser humano y su lengua nativa:

...native language is a powerful dimension of self-representation; it is one of the 'vital rings' of identity, separating us form others, affirming our uniqueness, yet confirming our membership in the `several larger rings of identity: of family, of region, of nation, of culture.[...]

For in truth, native language is the prime vehicle for the transmission of psychological and cultural essence, it is an ever-present embodiment of selfrepresentation, incorporating a view of the world and of ourselves, carrying a national-cultural epistemology 39 .

es, sin duda, una bella y emotiva manera de exponer la relación que existe entre el ser y el lenguaje humano, pero nos orientan poco sobre el origen que da lugar al fenómeno cuyas características describen; y que nos parece está en la influencia de lo social sobre lo personal. Influencia que se hace crítica en torno al fenómeno verbal, donde surge el interés por delimitar la relación, sin duda íntima, entre lengua, pensamiento y formas de manifestación externa del sujeto (o formas de conducta y auto-representación).

A este respecto quizás sea conveniente indicar que no solamente se pueden apreciar diferencias en esta relación, como las que Guiora 40 detecta en un determinado momento entre una comunidad que usa una lengua y otra; sino también, dentro de la misma lengua. Esto es lo que se manifiesta, por ejemplo, en la adopción de un tipo de código verbal u otro -como indica Berstein ${ }^{41}$ pero ello es debido, en último término, a las formas de interacción social, cuya manifestación más genuina es, sin duda, la expresión verbal.

\section{VI}

El interés principal de los trabajos de Guiora radica, a nuestro entender, en su aportación sobre el "set mental" 42 del sujeto en actividad verbal y en situación de incertidumbre -como la que implica la utilización de una lengua nueva ${ }^{43}$. Además de

39. GUIORA, A.Z. "Language, personality and culture”. En TESOL'81 Washington, D.C.1982. pág. 11.

40. GUIORA, A.Z. "Contrast validity and transpositional research: Toward an empirical study of psychoanalytic concepts”, Comprhensive Psychiatry, 13, 1972.

41. BERNSTEIN, B. Class, Codes and Control, vol. I, 1971, Londres, Routledge \& Kegan Paul.

42. Por ejemplo: "...the performance on the Thai pronunciation test (STP) reflects the same "mental set' necessary to accurately pronounce a second language." GUIORA, A.Z. y W.R. ACTON "Personality and Language behavior: a restatment" Languag Learning, 1, 1979, pág. 203.

43. Según este autor la "permeabilización de las fronteras del ego" creará las condiciones psicológicas apropiadas para una mejor adquisición de una nueva lengua. Las palabras siguientes merecen ser citadas en su totalidad por la analogía con los procedimientos sugestológicos: 
aportar evidencia sobre la importancia de los denominados "factores internos", las ideas de Guiora abundan sobre la hipótesis de que el proceso de asimilación de una nueva lengua puede constituir una tarea perturbadora de la configuración interna de la persona. Pero, sobre todo, sus formulaciones son enormemente sugerentes y apuntan hacia una dirección poco explorada por la investigación de la L2; lo que nos parece una deficiencia del propio marco teórico en el que se tratan estos aspectos y que se hace evidente en la realización de los correspondientes modelos adquisitivos que, en el mejor de los casos, no han ido más allá de conceptualizaciones a modo de "filtros afectivos"44 insertos en modelos desarrollados en la línea de la teoría de la infor-

"...the approach to a foreign language can be best made by recourse to the language ego paradigm. What is needed is a 'softening of the language ego boundaries, to make them more 'permeable'. The best analog can be found in the process of psychotherapy, In dynamic psychotherapy the lesson is learned that by confronting uncertainties, ambiguities, renunciations, and failures one will not need by force of necessity, lose the essential hallmark of human existence: mastery and autonomy. So it is with language learning. A patient and caring process of modelling, trail-identifications, temporary and reversible shifts in self representation can bring about a venturesome spirit of daring and psychological flexibility that coupled with the capacity to entertain cultural pluralism can open the door to the inner world of another language. A not incidental side benefit will be a boost in the learner's ability to entertain the alternative hypothesis on a cognitive level as well, significantly enriching his intellectual armamentarium." GUIORA, "Personality", op. cit. pág.11.

44. Son cinco las hipótesis que podemos entender comprendidas en la teoría de la adquisición de Krashen: "Acquisition vs. learning hypothesis", "The Monitor Hypothesis", "The Natural Order Hypothesis" "Input Hypothesis" y "The Afective Filter Hypothesis". La hipótesis de la existencia de unos mecanismos selectivos de la información ya había sido propuesta por Dulay y Burt (1977) en donde habla de "limitadores afectivos". La formulación posterior que hace Krashen incorpora el efecto de las variables afectivas -motivación, autoconfianza y ansiedad- como elementos que influyen sobre la "adquisición" de la lengua. Según Krashen dentro de su modelo adquisitivo existe un "filtro afectivo":

"The filter is that part of the internal processing system that subconsciously screens incoming language based on what psychologists call "affect"; the learner's motives, needs, attitudes, and emotional states. The filter appears to be the first main hurdle that incoming language data must encounter before it is processed further".

(DULAY,M.,H. BURT y M. KRASHEN, Language Two, Oxford, O.U.P.,1982, pág.46.)

Es generalmente admitido que cualquier modelo adquisitivo debe considerar la importancia de los factores afectivos en la asimilación de una lengua. Sin embargo, y a pesar del valor que tiene el desarrollo que Krashen efectúa sobre el concepto general de "lo afectivo", su configuración como "filtro" puede levantar serias dudas, aunque solamente sea por la escasa posibilidad que el mismo tiene de contar con una mínima realidad psicológica. A este respecto son pertinentes las palabras de Niesser:

"Formally speaking, every human being filter out cosmic rays, insect pheromones, and every other kind of information that does nos affect his behavior. Psychotically or biologically, however, this notion makes no sense. There is no mechanism, process, or system that functions to reject these stimuli such that they would be perceived if it were to fail. The perceiver simply does not pick them up,[...] Selection is a positive process, not a negative one."

(NIESSER, U. Cognition adn Reality, San Francisco, Freeman. 1976, pag. 79.)

Tanto el propio término "filter" como el modelo en el que está articulado constriñe un feómeno extremadamente complejo, como el de la asimilación de una lengua, dentro de los cauces del tratamiento de la información. Lo que, entre otras causas, lleva a la atribución -al menos implícitamente- de un papel pasivo al sujeto de aprendizaje. 
mación. Pero, si lo que buscamos es la competencia comunicativa, deberemos considerar las otras funciones que realiza el lenguaje verbal, además de la meramente informativa. A pesar de los problemas que de orden metodológico puede producir, el profesor de la L2 difícilmente se podrá beneficiar de la investigación sobre la adquisición si esta no le permite una visión suficiente del fenómeno adquisitivo. Quizás sería conveniente rebasar con más frecuencia los límites habituales de la investigación "normal" de la adquisición de la lengua. Entrar, como dice Lacan, en el ámbito de la palabra, ya que lo que desde el punto de vista de la información constituye redundancia, es resonancia en la palabra:

Pues la función del lenguaje no es informar, sino evocar. Lo que busco en la palabra es la respuesta del otro. Lo que me constituye como sujeto es mi pregunta ${ }^{45}$.

De manera que, el aprendizaje de una nueva lengua se vería, no como un fenómeno de "aprehensión" de un objeto ("adquisición"), sino como la forma en que el sujeto se modifica, se adapta estructuralmente ${ }^{46}$ a una nueva forma de poder hacer y representarse verbalmente. Porque adoptar una manera diferente de expresión verbal no parece un proceso innocuo, pues requiere un compromiso interno con la nueva tarea, que se muestra distinta a otros aprendizajes por implicar una nueva forma de auto-representación personal. Tanto es así, que el sujeto frecuentemente desarrolla formas de rechazo -de manera más o menos consciente. Una de sus manifestaciones, como hemos visto, puede ser la rigidez en la articulación verbal. En definitiva, la forma en que Guiora entiende la lengua: "I view language as a manifestation and as an engine of that intrincate and many-colored fabric we call personality" 47 ; sus tesis sobre los efectos perturbadores que la asimilación de la L2 puede tener para el sujeto y sus consideraciones al respecto nos parecen aspectos de sumo interés. La consideración debida de los mismos ayudaría completar aspectos no contemplados debidamente a la hora de formular un modelo adquisitivo de la L2.

...the task of learning a new language is a profoundly unsettling psychological proposition, not adequately described by limiting it to terms such as 'motivation', 'attitudes', or even 'affective variables'. What is required of the learner is not only a cognitive shift in terms of vocabulary, grammar, and syntax, but something much more formidable: the necessity to recategorize information according to the available and obligatory linguistic forms, task that inevitably must lead to a demand to assimilate alternative and new ways to describe and thus conceptualize, and ultimately experience, events in and around us. [...] And it is here that individual differences in the psychological defense system and flexibilities will be reflected in a capacity (or willingness) to attempt the shifting,

45. LACAN, J. Escritos, Méjico, Ed. Siglo XXI, 1981 pág. 117.

46. Esto es: autopoiéticamente, según sostienen Maturana y Varela (1980), op.cit.

47. GUIORA, “The dialectic”, op. cit., pág. 9. 
to dare the recategorization and the ensuing reconceptualization and re-experiencing, to embrace the "alternative hypothesis" without fear of loosing the grip on the psychological integrity for which native language serves as such a powerful anchor ${ }^{48}$

Lo que Guiora no parece contemplar, sin embargo, es que existen otras variables relacionadas con su "alternative hypothesis" y que, no obstante, no podemos considerarlas directamente relacionadas con los fenómenos de reconceptualización, recategorización, etc., (o cualquier otro concepto cognitivo). A pesar de ello, sin embargo, pueden afectar tanto a la actividad verbal con la L1, como con cualquier otra lengua que el sujeto emplee. Estas otras variantes, al ser igualmente generadoras de tensión y conflictos internos al sujeto, tienen, también, un efecto inhibidor de la adopción de esta "hipótesis alternativa". Nos referimos a los efectos derivados de la inconscientemente asumida actitud del "otro". Pues, si bien es cierto que la lengua es el medio por excelencia de nuestra forma de auto-representación, no es menos cierto que, como dice Halliday, "a language is a form of belonging" 49 . Este sentimiento de pertenencia y, por lo tanto, de comunión en la significación, no es un sentimiento pleno fuera del ámbito de los primeros años de vida, cuando se nos da por exceso "anticipatory illusion"- y gracias a lo cual podemos adoptar la lengua de nuestros mayores. Después, debemos confirmarla constantemente -y, desafortunadamente, no siempre con éxito ${ }^{50}$.

\section{VII}

¿Cuál será el sentimiento de pertenencia de un alumno que da sus primeros pasos en el aprendizaje de una lengua extranjera?, podemos preguntarnos. En las sociedades que cuentan con un determinado número de miembros diglóticos es fácilmente constatable la resistencia que encuentran las personas que no lo son

48. GUIORA, "Language”, op. cit. pág. 8.

49. A este respecto GUIORA, "The dialectic", op. cit., pág. 3, se manifiesta inequívocamnte en el mismo sentido:

"...native language is a powerful dimension of self-representation: it is one of the 'vital rings' of identity, separating us from others, affirming our uniqueness, yet confirming our membership in the "several larger rings of identity: of family, of region, of nation, of culture."

Otra cosa son las soluciones concretas que de sus ideas pueden derivarse y que, a nuestro entender, están limitadas a un tratamiento individual del problema.

50. La sociolingüística ha abstraído este fenómeno al buscar causas en los conflictos dialectales, o de códigos, para explicar, por ejemplo, la parte del fracaso escolar atribuible a cuestiones linguísticas -teoría del déficit, stereotype hypothesis, teorİa de los códigos, etc. En un nivel más concreto tenemos el efecto Hothorne, o efecto Pigmalión, esto es, la tendencia que tenemos a comportarnos de acuerdo a lo que se espera de nosotros, etc. 
para que sea aceptado el uso de su L2 como forma "natural" de comunicación en el grupo, por parte de los otros. Esto se produce aun cuando su nivel de competencia comunicativa pueda considerarse suficiente para mantener el intercambio verbal, y puede servir de ilustración de lo que queremos decir al afirmar que no se trata sólo de un problema cognitivo ${ }^{51}$. Pero, si la adquisición de la L2 es entendida como algo más que un fenómeno cognitivo, si tenemos presente las siguientes palabras:

Una lengua es un vínculo de unión y de solidaridad entre los que la hablan, a la vez que segrega a quienes la desconocen. "Bárbaro", término utilizado para designar a quiénes no hablan la lengua de un pueblo, significa sencillamente "el que habla otra lengua". Toda comunidad tiene como propia su lengua y la transmite en tanto existe como comunidad. 52

De nuevo aparecen en lo inter-individual las causas de, al menos, buena parte de lo que puede presentarse como intra-individual y, por lo tanto, la necesidad de considerar las circunstancias de tipo social que dentro del aula pueden llevar a una configuración psicológica del alumno facilitadora del aprendizaje de la nueva lengua. No se conseguirá, a nuestro entender, con ejercicios de autorepresentación la permeabilidad al "ego verbal" de Guiora, por importante que pueda esto parecer; por el contrario nos parece más necesario crear las condiciones externas en las que este ejercicio verbal sea aceptado de forma "natural" por el alumno y los otros miembros del conjunto micro-social que componen el aula de idiomas. No proponemos, pues, un mero ejercicio formal; sino que toda la actividad esté enfocada a satisfacer apropiadamente algo que, por otra parte, constituye una necesidad de "acoplamiento estructural" auténticamente humana: la comunicación, pero en este caso en la nueva lengua. Esto requiere articular la actividad didáctica con una realidad psico-social dentro del aula, para que no produzca en los alumnos el sentimiento de ser "bárbaros" imitando una forma de expresión verbal que se siente fuera de la extensión que los límites de nuestro ego permiten. Es imprescindible el máximo respeto de toda actividad en el aula a las variaciones personales del alumno. Una enorme dificultad 53 .

51. También es evidente un determinado grado de inhibición al usar la L2 con aquellos con los que anteriomente se ha sostenido la comunicación en otra lengua. Los constantes cambios de lengua en las conversaciones cruzadas son demasiado frecuentes y significativos para que sea necesario insistir.

52. SERRANO, S. La Semiótica. Barcelona, Montesinos, 1981, pág. 61.

53. Para un análisis de estas dificultades y propuesta de posibles soluciones extraídas de la experiencia personal del autor a este respecto, ver : Alcántara , F. 1984 La Sugestopedia: su influencia sobre los factores internos que intervienen en el aprendizaje de la L2 S.P.U.C.B. Barcelona. 


\section{VIII \\ A modo de conclusión: La persona y las nuevas tecnologías}

El aprendizaje de una lengua en un entorno de instrucción formal suele hacer más evidentes algunos de los aspectos negativos que sobre el aprendizaje verbal tiene esa vinculación entre la persona y la lengua, asunto sobre el que hemos reflexionado ampliamente. Creemos que esta vinculación debe ser debidamente considerada por el profesor de L2, tanto en el momento de la planificación de su actividad, como a la hora y manera de realizar lo planeado. En ambos casos se requiere una buena consideración del uso de los recursos didácticos disponibles, o deseables por su conveniencia.

La aplicación de los recursos que las nuevas tecnologías permiten hoy es, a nuestro entender, altamente deseable, pues éstos posibilitan el cambio de la naturaleza del objeto de aprendizaje convencional, así como la actividad orientada al mismo; también pueden modificar convenientemente los papeles tradicionales, tanto del profesor como el de los alumnos. Se ha dicho con insistencia que para reformar la enseñanza se necesita cambiar la manera de participar el profesor y el alumno en el proceso didáctico. La nueva situación exigiría, quizás, un profesor más dedicado a "facilitar" el aprendizaje; y un alumno con mayores posibilidades de asumir directamente el ritmo, manera y responsabilidad de su aprendizaje. Posiblemente así le sea más fácil asumir esa actitud participativa que de él se espera en el aula.

Deutsch ${ }^{5}$ contrasta tres posibles formas de actuar el alumno en el aula: la individualista, cuando no tiene en cuenta ningún tipo de vinculación con las necesidades de los otros; la competitiva, que puede tener buenos rendimientos pero, a su vez, crear grandes frustaciones; y la cooperativa o participativa, en la que, idealmente, cada logro del alumno contribuye a la consecución de los objetivos que pretenden los demás. Esta ha sido entendida casi siempre por la mayoría de los profesores de L2 como la actitud más deseable pero, también, considerada la de mayor dificultad -no sin razón. No es necesario detenerse aquí en los obstáculos que han de salvarse para lograr que el alumno mantenga una actitud participativa y corresponsable de su aprendizaje; tampoco -por bien conocida- hablaremos sobre la sobrecarga que supone para el profesor el desarrollar su acción didáctica en esta línea; sí nos parece conveniente, sin embargo, volver al pensamiento de Vigotsky, de nuevo, para ver como la acción cooperativa es condición esencial en el aprendizaje y cómo puede ser llevada a cabo la acción colaboradora con ayuda, no ya de los sistemas expertos informáticos con los que se ha pretendido enseñar lenguas, sino con el apoyo de entornos hipermedia, cuyo uso apropiado puede propiciar la actividad colaboradora y , consecuentemente, el aprendizaje.

54. Deutch, M. 1949 A theory of co-operation. Human Relations, 2(2), 129-153 
Quizás convenga recordar primero la importancia de la acción cooperativa en el mismo origen y en la posterior evolución del lenguaje verbal, según nos dice Malinowski55. Después, considerar que para Vigotsky la acción cooperativa con el niño constituye un elemento esencial de su desarrollo, pues contribuye positivamente a su crecimiento intelectivo y a su desarrollo verbal. El concepto vigotskiano de "zona de desarrollo próximal" gravita precisamente sobre la diferencia que puede establecerse entre lo que es capaz de hacer el niño por sí mismo, y lo que logra con la cooperación del adulto 56 .

La organización social humana permite que las condiciones sean idóneas para que la acción cooperativa se mueva casi siempre dentro de esa zona en el proceso de adquisición de la L1; no suele ser así en el caso del aprendizaje de otras lenguas. En este último caso la acción conjunta que puede establecerse entre el hablante competente y el que está aprendiendo no siempre es "cooperativa" o "colaboradora" -en el sentido que estamos dando aquí a estos términos- no se acerca a la "zona próximal” y, por lo tanto, difícilmente producirán un efecto positivo sobre la adquisición. Por el contrario, puede tener efectos negativos e inhibir ejecuciones posibles y propias de niveles de competencia ya alcanzados cuando la situación se aleja considerablemente de lo ideal con respecto a estos factores. Como consecuencia de la vinculación entre la lengua y la persona, las carencias en la representación verbal de la persona del alumno necesita un suplemento en el grado de colaboración del "otro" que, con cierta frecuencia, no encuentra. De ahí buena parte de la dificultad de aprendizaje de otras lenguas a partir de cierta edad, cuando el grado en empatía del interlocutor y su disposición colaboradora es menor. Sin embargo, se ha comprobado que el desarrollo del nivel de interlengua del alumno es mayor y más rápido si éste se mueve cooperativamente en un umbral similar al de la "zona de desarrollo próximal" de Vigotsky (Py, 1989)57 para lo que es necesario, no solamente una tarea a realizar de dificultad apropiada, sino que es altamente deseable saber que se cuenta con la cooperación del interlocutor en la resolución de la misma.

El aprendizaje cooperativo parece ser la alternativa ideal a los sistemas expertos, pues en lugar de intentar transmitir el conocimiento lingüístico, lo que parece ser más conveniente es ofrecer al alumno de L2 unas condiciones -las que puede ofrecer un determinado entorno informático- en las que progrese en su proceso adquisitivo por medio de la ejecución de diversas actividades cooperativas que el entorno ofrece. La

55. Malinowski, b. 1935 Coral Gardens and their Magic. Vol.II. G. Allen@ Unwin, Londres.

56. Esta zona puede tener en el aprendizaje de una lengua un papel equivalente al telón afectivo anteriormente mencionado y, desde luego, puede ser el nuevo "sistema de apoyo" del que nos habla Bruner.

De todas formas, quizás no sea inútil recordar que la naturaleza espacial de estos conceptos es meramente metafórica cuando decimos que la zona de "desarrollo proximal" es la distancia entre el potencial comunicativo del alumno, en aspecto concreto y en una lengua determinada, y lo que puede expresar en un momento dado con la ayuda del profesor.

57. Py, B. 1989. L'acquisition vue dans la perspective de l'interaciton. En DRLAV, 41, 83-100. 
capacidad de los ordenadores personales aumenta constantemente, por lo que cada vez es posible modelar con mayor precisión aquellos aspectos que podemos suponer facilitadores de la adquisición de la lengua objeto de aprendizaje. Las distancias de tipo intelectivo entre el ser humano y la máquina no suponen, en principio, un gran incoveniente para el usuario del ordenador personal quien, como puede fácilmente comprobarse, es capaz de suponer capacidad interactiva verbal al ordenador y parece considerarlo un apoyo deseable cuando siente que el entorno interactivo que se le ofrece está dotatado del suficiente nivel de desarrollo. Es interesante destacar, sobre todo, que el alumno reacciona muy positivamente con respecto a lo que podemos denominar "neutralidad psicológica" del instrumento de interacción que se le ofrece. De forma que, por medio del entorno hipermedia, no solamente puede contar el que intenta aprender otra lengua con una fuente de información abundante y de paciencia inagotable para informar una y otra vez sobre lo ya anteriormente dicho, sino que sobre este entorno el alumno puede actuar experimentalmente a través de un circuito interactivo en el que la respuesta del "otro" es determinista y cooperadora por principio.

Esta relación instrumental hombre-máquina no tiene que circunscribir la actividad total del alumno al ámbito de la actividad estrictamente individual, por el contrario, la colaboración con otros alumnos puede facilitarse de esta forma -al tener mejores posibilidades de ampliar la capacidad de relación en la lengua objeto de adquisición. Probablemente de esta manera quede superada parte de la dificultad de alcanzar los niveles previos a la actividad interindividual en la lengua nueva, sin que los participantes se sientan extraños en su forma de representarse. La interacción verbal cooperativa entre compañeros se potenciaría así y, quizás, el paso de lo interindividual a lo intraindividual, según dice Vygotski, se facilite.

No cabe duda de que la conveniencia de este enfoque no puede mantenerse más que en un nivel teórico, pues se carece de experimentación suficiente; la escasa que se ha realizado ha estado fundamentalmente orientada al aprendizaje en general58 $\mathrm{y}$, en todo caso, el tipo de interacción previsible estará limitada a aspectos tanto gramaticales como pragmáticos concretos, dentro de un ámbito muy restringido de la lengua objeto $^{59}$. Sin embargo, si algunos de los programas que se se encuentran en fase de desarrollo en este momento consiguen los objetivos propuestos, las posibilidades de ese entorno cooperativo serán sustancialmente mejores ${ }^{60}$. Se hará necesario, no obs-

58. Véase Chan (1993). Sobre la incidencia sobre la interacción en el aula son reveladores los trabajos básicos de Tharp et. al.( 1988).

59. C.f.Renié and Charnier (1995) quienes han desarrollado un entorno cooperativo para facilitar la adquisición de las interrogativas directas del francés.

60. Nos referimos concretamente al desarrollo de la denominada "hypermedia technology", términos nuevos con los que se describe los interfaces de un programa hipertexto con sistemas de gestión de información auditiva y visual, esta última en forma de gráficos, fotografías, vídeos, texto, etc. 
tante, su adecuación previa, en el caso de proyectos como SECC61, y en todo caso la elaboración de materiales didácticos apropiados.

Cabe mencionar también que, dentro de este mismo campo de ideas, la teoría de Guiora encuentra su correlato y posible explotación. Este es el caso de las conclusiones deducibles de los trabajos de Brazil (1992)62 sobre la pronunciación de una lengua extranjera, su proyección sobre la sintaxis y sobre el valor actitudinal, Sobre estas bases Allen (1993)63 ha desarrollado un entorno hipermedia destinado a mejorar la entonación, donde el alumno pude acceder a multitud de medios, tanto visuales como sonoros; textos, ejemplos, ejercicios de autoevaluación, etc. desde cualquier punto del sistema; y todo susceptible de poder ser utilizado conjuntamente con otro alumno o en trabajo individual. Este sistema, como otros existentes sobre lectura (por ejemplo Randall, et. al (1994)64, composición guiada (Brehony et. al 1994)65, etc, no carece de limitaciones que deben tenerse en cuenta al considerar la idea general que puede generar lo aquí dicho. No obstante, el desarrollo que las bases de datos hipermedia están últimamente teniendo -véase por ejemplo Yoshitaka (1995)66- permite suponer que buena parte de esas limitaciones desaparecerá en los próximos sistemas.

\section{Resumen}

Hemos intentado desarrollar en este trabajo aspectos que pueden parececer bien diferentes, pero desde luego importantes y, a nuestro entender, convergentes: el concepto psicoanalítico de "persona", la lengua, su aprendizaje y las nuevas tecnologías.

Según indicamos al comienzo, si hay algo que puede caracterizar bien a la actividad humana en el siglo XX es el tremendo avance que ha conseguido en el conocimiento del entorno físico: lo exterior al hombre. Otra cosa bien diferente puede decir-

61. El proyecto SECC comenzó en noviembre de 1993 y se acabará dentro del presente año 1995. Participan en el empresas como Siemens-Nixdorf, Cap Gemini, Alcatel Bell, universidades europeas y americanas, etc. El objetivo general del mismo no es propiamente didáctico, sino que esta orientado principalmente a su explotacion ofimática. Persigue concretamente el desarrollo de una herramienta para ser utilizada por el escritor te textos técnicos dentro de una variedad de inglés simplificado. Sin embargo el sistema será capaz de comprobar el documento en su totalidad, tanto en sus aspectos sintácticos como léxicos. Se dedicará una atención especial a las dificultades que los escritores no ingleses puedan encontar en la creación de este tipo de documentos y podrá realizar correcciones a nivel textual. No cabe duda que será suceptible de ser adaptado para su utilización en el aula, y en todo caso será un modelo para el desarrollo de sistemas similares que funcionen en distintos niveles de interlenguas.

62. Brazil, D. 1992. Advanced English Pronunciation. Cambridge University Press, Cambridge.

63. Allan, M. 1993. "Teaching, Text and Technology. A Hypermedia Environment". En M. Baker et al. (eds) Text and Technology. J. Benjamins, Amsterdam.

64. Randall, M, et al. "Reading is creative, too!.” Computer Assisted Language Learning. 7,3.

65. Brehony, T., K. Ryan 1994. "Francophone stylistic grammar” C.A.L.L. 7,3.

66. Yoshitaka, A. et al. 1994. "Knowledge-assisted content-based retrieval for multimedia databases". En I.E.E.E. Multimedia. 1,4. 
se de los logros alcanzados en la comprensión de lo interior del ser humano, lo que genéricamente podemos llamar lo mental. La mente y sus manifestaciones -la verbal es sin duda la más preclara- se han resistido a un grado similar de conocimiento. La inmensa extensión del campo de lo verbal ha requerido la especialización modular en sub-campos; es posible que esta situación haya potenciado concepciones de la capacidad verbal como algo que existe en considerable independencia de otras manifestaciones internas del hombre, formando así módulos relativamente autónomos, algunos concebidos como innatos. Los que esto sostienen tienden a explicar los fenómenos verbales por sí mismos, desligados de las circunstancias internas del sujeto que los produce. Desde una postura opuesta se entendería, por el contrario, que todo lo verbal es parte inseparable de la actividad mental, de la actividad interna en la que la manifestación verbal es sólo una parte. Consecuentemente el aprendizaje de una segunda lengua se entendería siempre en función de lo que posibiliten las condiciones internas del alumno. Una manifestación de ellas es la configuración de su forma de representarse ante los otros. Persona y lengua parecen pues dos aspectos fundamentales, las maneras posibles de optimizar la relación entre ellos en el aprendizaje de la L2 resultan de extremo interés y la moderna ciencia de la información parece tener mucho que aportar a este respecto. 